\title{
Structure of the Cyanobacterial NAD(P)H Dehydrogenase-Like Complex of Oxygenic Photosynthesis
}

Thomas G. Laughlin ${ }^{1,2}$, Andrew N. Bayne ${ }^{3,4}$, Jean-François Trempe ${ }^{3,4}$, David F. Savage ${ }^{1}$ and Karen M. Davies $^{1,2 *}$

${ }^{1 .}$ Department of Molecular and Cell Biology, University of California, Berkeley, CA, USA.

2. Molecular Biophysics and Integrative Bioimaging Division, Lawrence Berkeley National Laboratory, Berkeley, CA, USA.

3. Groupe d'étude des protéines membranaires (GEPROM), Montréal, Quebec, Canada.

4. Department of Pharmacology and Therapeutics, McGill University, Montréal, Quebec, Canada.

* Corresponding author: KMDavies@lbl.gov

Conversion of carbon dioxide $\left(\mathrm{CO}_{2}\right)$ into sugar is one of the most important metabolic pathways for the survival and propagation of nearly every organism on earth. Although confined to only a subset of species, $\mathrm{CO}_{2}$ fixation provides the food, energy and basic building blocks for the formation of nearly every biomolecule in the world. In plants, green algae and cyanobacteria, the energy required to drive the formation of sugar from $\mathrm{CO}_{2}$ is obtained from the sun, whose light rays excite electrons in the "green" chlorophyll molecules. These excited electrons leave the chlorophyll molecule and start a long complex journey through the thylakoid membranes until they reach and reduce the energy storing biomolecule nicotinamide adenine dinucleotide phosphate $\left(\mathrm{NADP}^{+}\right)$. Furthermore, as they partake in their journey, protons are pumped across the membrane which ultimately power the formation of a second energy storing biomolecule, adenosine triphosphate (ATP). Both ATP and NADPH are required to power the conversion of $\mathrm{CO}_{2}$ into sugar, however, the ratio produced by a single passage of electrons from chlorophyll to NADP ${ }^{+}$ is not optimal for $\mathrm{CO}_{2}$ fixation and additional ATP is required. This additional ATP is thought to be generated in part by the electron coupled proton-pumping activities of NAD(P)H dehydrogenase-like Complex (NDH) - the complex I-like enzyme of oxygenic photosynthesis.

The NAD(P)H dehydrogenase-like complex $(\mathrm{NDH})$ is a $0.42 \mathrm{MDa}$ multi-subunit enzyme related to the NADH dehydrogenase complex of the oxidative phosphorylation pathway. It contains 11 of the 14 core subunits from the respiratory enzyme as well as several new subunits specific to the photosynthetic complex. One of the key questions regarding NDH function is how does this complex obtain electrons from the photosynthetic pathway and redirect them back to the plastoquinone pool. This conundrum arises because the three subunits responsible for accepting electrons from NADH in the respiratory enzyme are missing from the photosynthetic enzyme. To gain a more in-depth understanding of how NDH obtains electrons in the photosynthetic pathway and the role of the additional subunits in this process, we have determined a $3.1 \AA$ and a $3.6 \AA$ structure of the detergent-solublized NDH from the thermophilic cyanobacteria Thermosynechococcus elongatus using single particle cryoEM [1].

In both of our structures, the 11 core subunits and the 7 photosynthetic specific subunits are clearly identifiable allowing the generation of an atomic model (Figure 1a). Of particular interest was subunits $\mathrm{NdhS}$ and NdhO which have identical SH3 folds and are located either side of a very basic $\beta$-hairpin loop of NdhI, which is positioned directly above the first 4FeS ion cluster of the electron transfer centers. SH3 folds are often found in ferredoxin binding proteins and act to direct ferredoxin to the site of electron transfer. The diameter of ferredoxin $(25 \AA)$ matches the distance between the two SH3 subunits, and the 
first 4FeS cluster, suggesting the possibility of two ferredoxin binding sites on NDH (Figure 1B). This prediction is further supported by two positively charged patches in the $25 \AA$ gaps and the basic $\beta$-hairpin loop of NdhI which is necessary to counteract the highly negative surface charge of ferredoxin (Figure 1C). The possibility of having two ferredoxin binding sites is particularly significant for redox chemistry as ferredoxin can only carry one electron at a time, but plastoquinone requires two electrons to become fully reduced. Thus, two binding sites would greatly reduce the chances of a long-lived partially reduced plastoquinone which is highly reactive.

Other significant findings in our structure were: 1) a fourth unidentified electron dense patch on the side of the cytoplasmic arm, which might act as an addition electron accepting site and 2) a glycolipid and $\beta$ carotene molecule at the interface of $\mathrm{NdhD}$ and $\mathrm{NdhF}$ which may function in excess energy dissipation as observed in other photosynthetic molecules. All three finding provide the structural foundations for mutation studies to investigate the mechanism of electron acquisition and transfer by NDH.

The data present in this paper was recently published in [1].

\section{References:}

[1] TG Laughlin et al., Nature 566 (2019), p. 411.

[2] We thank the Yano/Yanchandra lab for T. elongatus membranes, specimen screening and data acquisition was performed at the Donner cryoEM resource and on the BACEM KRIOS. EM training and computation support was provided by E. A. Montabana, D. Toso and P. Tobias. Advice on model building was provided by T. H. D. Nyugen and B. J. Greber, and NDH biology by the Savage lab and N. Fisher (MSU). Funding: D.F.S. and K.M.D were supported by the US Department of Energy Grant DESC00016240 and DE-AC02-O5CH11231, respectively. T.G.L. was supported by a National Science Foundation Graduate Research Fellowship and The Molecular Basis of Cell Function NIH predoctoral training grant (NIGMS project 5T32GM007232-38).

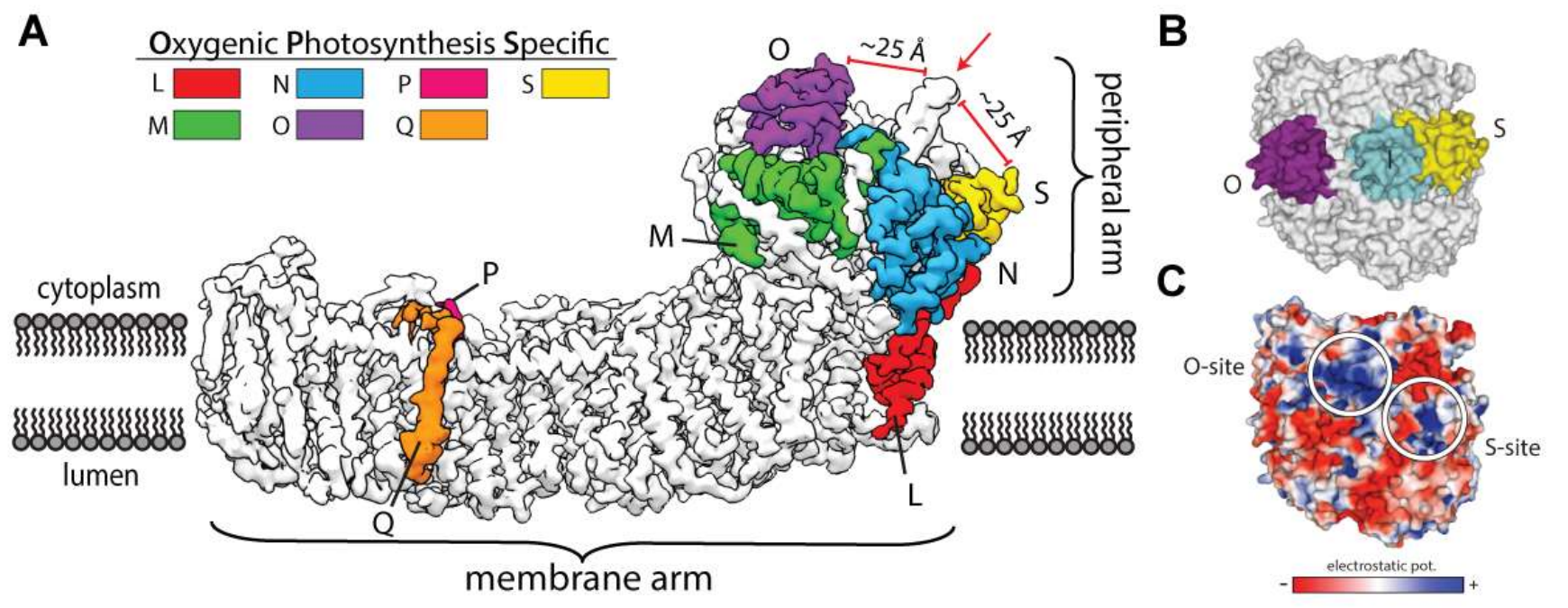

Figure 1. A) Structure of NDH from T. elongatus determined by single particle cryoEM. The seven photosynthetic specific subunits of NDH are highlighted in color. The $\beta$-hairpin loop of NdhI is indicated by the red arrow. The $25 \AA$ gaps between the hair pin loop and $\mathrm{NdhS}$ and $\mathrm{O}$ is sufficient to accommodate a ferredoxin molecule. B) Top down view of cytoplasmic arm indicating positions of NdhS, O and I. C) electrostatic surface of B indicating basic patch which could facilitate ferredoxin binding. 